Tropical Journal of Pharmaceutical Research October 2020; 19 (10): 2235-2241

ISSN: $1596-5996$ (print); 1596-9827 (electronic) (C) Pharmacotherapy Group, Faculty of Pharmacy, University of Benin, Benin City, 300001 Nigeria

\title{
Protective effect of Salvia miltiorrhiza in rheumatoid arthritis patients: A randomized, single-blind, placebo- controlled trial
}

\author{
Zheng Yuan, Changsong Zhao, Qiang Zhang*, Zhengrong Gao \\ Department of Orthopedics, Beijing Ditan Hospital, Capital Medical University, No.8, Jingshun East Street, Chaoyang District \\ 100015, Beijing, China
}

*For correspondence: Email: zhangqwte@sina.com; Tel: 0086-010-84322527

Sent for review: 24 February 2020

Revised accepted: 23 September 2020

\begin{abstract}
Purpose: To study the protective effect of Salvia miltiorrhiza (SM) against rheumatoid arthritis (RA) in RA patients.

Methods: Sixty RA patients were divided into two groups: $S M(n=30)$ and placebo $(n=30)$ groups given SM at a dose of $250 \mathrm{mg} / \mathrm{kg}$ (3 capsules/day), and placebo (3 capsule/day), respectively, for 12 weeks. Patient responses based on American College of Rheumatology (ACR), health assessment questionnaire (HAQ) score, and global assessment of disease (GAD) were recorded. Moreover, Disease Activity Score (DAS) 28, pain score (visual analogue score, VAS), rheumatoid factor (Rh factor), and inflammatory cytokines (markers) were determined.

Results: After 12 weeks of intervention with SM, ACR20 (30\%)/ACR50 patient response (13.3\%, i.e. score for swelling and tenderness of joints), was significantly improved. There were considerable reductions in GAD, HAQ, DAS 28, pain score (VAS), and levels of erythrocyte sedimentation rate $(E S R)$, acute phase reaction protein (CRP), Rh factor (IgM) and inflammatory cytokines (IL-1 $\beta, I L-6$ and $T N F-\alpha)$, when compared to placebo $(p<0.01)$. Treatment with SM produced milder adverse effects than treatment with placebo $(p<0.01)$.

Conclusion: Overall, $S M$ produces better anti-RA effect than placebo by significantly altering ACR patient response, reducing GAD, HAQ, DAS 28 scores, Rh factor, ESR, CRP and inflammatory cytokines in RA patients. However, a large-scale clinical trial is needed before SM can be recommended for combating $R A$ and its related symptoms.
\end{abstract}

Keywords: Salvia miltiorrhiza, Rheumatoid arthritis, DAS 28, Adverse effect

\begin{abstract}
This is an Open Access article that uses a fund-ing model which does not charge readers or their institutions for access and distributed under the terms of the Creative Commons Attribution License (http://creativecommons.org/licenses/by/4.0) and the Budapest Open Access Initiative (http://www.budapestopenaccessinitiative.org/read), which permit unrestricted use, distribution, and reproduction in any medium, provided the original work is properly credited.
\end{abstract}

Tropical Journal of Pharmaceutical Research is indexed by Science Citation Index (SciSearch), Scopus, International Pharmaceutical Abstract, Chemical Abstracts, Embase, Index Copernicus, EBSCO, African Index Medicus, JournalSeek, Journal Citation Reports/Science Edition, Directory of Open Access Journals (DOAJ), African Journal Online, Bioline International, Open-J-Gate and Pharmacy Abstracts

\section{INTRODUCTION}

Rheumatoid arthritis (RA) is a chronic and destructive auto-immune disease characterized by increased inflammation of the joints and adverse effects on movement. The major symptoms of RA are swollen joints, arthralgia (joint pain), and stiff joints due to damage to cartilage and bone of joints, especially at the knees, ankles, feet, and elbow [1]. The disease affects about $0.5-2 \%$ of the global population, and is associated with high morbidity (deformity 
and disability), especially amongst elderly women [2]. The quality of life of RA patients is considerably hampered due to disability and deformity. Hence, most RA patients need extensive care and assistance even in the performance of basic day-to-day activities, leading to serious economic burden (absence from work) [3].

The pathophysiology of RA is still not fully understood. However, oxidative stress (excessive free radical generation), altered immune response and inflammatory events are considered as pivotal events in the pathogenesis of RA [1]. Currently, disease-modifying antirheumatic drugs (DMARDs), non-steroidal antiinflammatory drugs (NSAIDs), and steroids are recommended for the treatment or management of RA and its related symptoms. These treatments are effective only at the early stages of the disease, but they are expensive in the long run, and they also result in serious adverse reactions, apart from being ineffective in some RA patients [4]. Therefore, many researchers are shifting their focus to Traditional or Complementary or Alternative medicine which have lower adverse effects, and are more affordable and more effective in most RA patients [5].

Salvia miltiorrhiza (SM) Bunge is a well-known Traditional Chinese Medicinal (TCM) plant (Danshen/Reg sage) which belongs to the family Lamiaceae. The dried roots are recommended for treatment of cold, fever, nausea, joint pain and sprain, and for enhancement of blood circulation [6]. The dried roots of SM rhizome are rich in diterpenoids (tanshinone), phenolic compounds (salvianolic acid A/B), and flavonoids [7]. Salvia miltiorrhiza (SM) has a wide spectrum of therapeutic and beneficial properties such as anti-inflammatory, immunosuppressive, antioxidant, anti-tumor, pro-apoptotic, antidiabetic, anti-rheumatoid arthritis, as well as cardio-, hepato- and reno-protective effects. These properties are due to the presence of various bioactive phytocomponents such as tanshinones (I, IIA/IIB), salvianolic acid $A / B$, and lithospermic acid [8]. Previous studies on cell lines and animal models have revealed that SM and its bioactive components (tanshinone and salvianolic acid) possess potent antiinflammatory, immune-suppressive, antiadhesion and anti-rheumatoid arthritis properties [9-11]. Nevertheless, to date, no clinical trials have been conducted with SM in RA patients. Hence, in the present investigation, a singleblinded and randomized placebo-control clinical trial was conducted to study the protective effect of SM in RA patients.

\section{EXPERIMENTAL}

\section{Recruitment of subjects and ethical approval}

This single-blind, randomized placebo-controlled clinical trial was conducted by initially recruiting 78 RA patients (outpatients) aged between 22 and 72, based on American College of Rheumatology (ACR) criteria and Disease Activity Score (DAS 28) > 5.1, with the help of flyers/posters and newspaper advertisements. All patients underwent basic physical examination, vitals and biochemical parameters to cross-check their health status and wellness. Moreover, information was obtained especially about their medication before enrolling the participants for this trial. The inclusion criteria covered only RA subjects (determined based on ACR criteria) with at least 5 swollen joint/pain score, and who were on DMARDs. Heavy smokers or alcohol drinkers, drug addicts, cancer patients, and patients who had hepatic, cardio-pulmonary, gastro-intestinal or renal disorders/diseases, were excluded. Moreover, subjects allergic to plant products and other auto-immune disorders were not included. Based on these exclusion and inclusion criteria, only 60 RA subjects were finally enrolled in the trial.

This clinical trial was conducted at Beijing Ditan Hospital Capital Medical University from March to December 2018, and was approved by the ethical clearance board members of Beijing Ditan Hospital of Capital Medical University, China (approval no. $=$ TMBD-1546/12-2017). All the procedures used in this trial were conducted in accordance with the guidelines of the Declaration of Helsinki [4]. All the 60 RA subjects enrolled in this study were asked to sign a consent form before explaining the details of this trial. The flow chart of the clinical trial is shown in Figure 1.

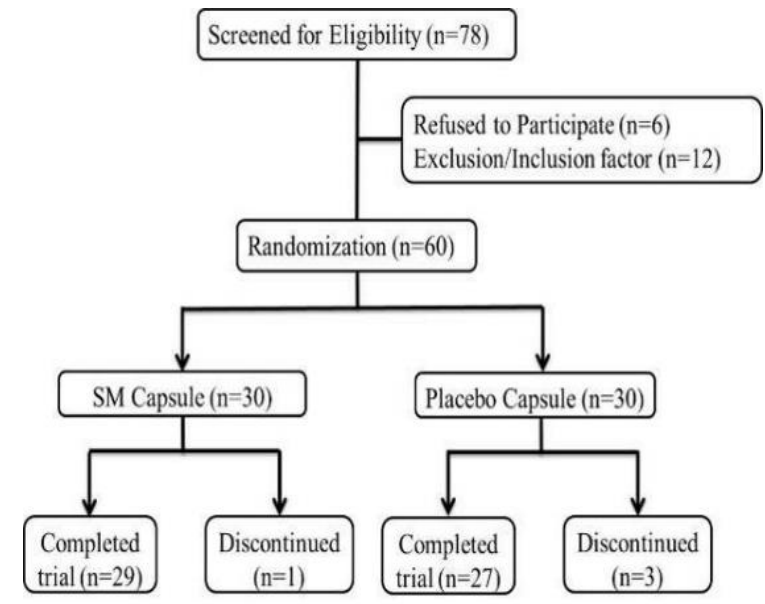

Figure 1: Flow chart of this clinical trial 


\section{Experimental protocol/intervention}

The 60 RA patients were randomly divided into two groups: SM $(n=30)$ and placebo $(n=30)$ groups, using computerized random digital numbers. Each participant in SM group was given 3 capsules every day at a dose of 250 $\mathrm{mg} / \mathrm{kg}$ for 12 weeks. The SM/Danshen extract and placebo capsules were provided by Wuxi Gorunjie Natural-Pharma Co. Ltd, Wuxi, China. The dose of SM was chosen based on previous studies conducted by van Poppel et al [12]. There were no differences between the SM and placebo capsules in terms of color and flavor. The placebo was filled with starch, and it had $1 \%$ SM which gave it a flavor similar to that of SM.

\section{Sample collection and measurement of various parameters}

Basic medical checkup covering all the vitals were done to confirm the health status. Then, blood samples were collected by a phlebotomist, at baseline and at the $12^{\text {th }}$ week (end of the study). The blood samples were kept at $-80{ }^{\circ} \mathrm{C}$ prior to analysis. Serum samples were obtained after centrifugation of clotted whole blood, and subjected to various biochemical analyses. Peripheral blood mononuclear cells (PBMCs) were separated from whole blood using ultracentrifugation technique, based on the Salunkhe method [13]. The PBMCs were subjected to rheumatoid (Rh) factor-like IgM analysis using immune-nephelometry with BNII analyzer kits (Siemens Medical Solutions Diagnostics; Munich; Germany) based on the manufacturer's protocol. Erythrocyte sedimentation rate (ESR) was calculated using Westergren method. Serum high sensitivity C reactive protein (hs-CRP) was determined using commercial ELISA CRP kit (MYBioSource; CA, USA). Various inflammatory markers, viz, interleukin 1 beta/6 (IL-1 $\beta / 6)$ and tumor necrosis factor-alpha (TNF- $\alpha)$, were quantified with commercial (Quantikine) ELISA kit (R\&D System, MN, USA) method based on manufacturer's instruction.

Other major factors such as those associated with global assessment of disease (GAD, on a scale of 0 - 100), including core disability Health Assessment Questionnaire (HAQ) score/index (score range of $0-3$, with 20 different questions on dressing, walking, grooming, eating and rising) were calculated [14,15]. Moreover, ACR criteria i.e. ACR 20/50 patient response (positive improvement) which refers to reduction of 20 or $50 \%$ in the number of swollen and tender joints; patient pain (VAS), hs-CRP, as well as GAD and HAQ were determined with DAS 28-ESR (including 28 joints/tender-radiographic examination, ESR, and VAS pain scale of 0 $100)$ by a trained rheumatologist and physician, at baseline and at the $12^{\text {th }}$ week $[16,17]$. Finally, the incidence of various adverse or side effects such as headache, anemia, dizziness, nausea/vomiting, dyspepsia, anorexia, diarrhea, insomnia, and skin rashes/itching were recorded after 12 weeks of intervention.

\section{Statistical analysis}

Changes in the various parameters after the intervention (at the $12^{\text {th }}$ week of the study) between the placebo and SM groups were analyzed using a one-way ANOVA and Student's $t$-test, while intra-group comparisons between baseline and $12^{\text {th }}$ week in each group (SM or placebo) were conducted using paired $t$-test. All statistical analyses were carried out with SPSS software (ver 21 from IBM Corp, CA, USA). A probability value of $<0.05$ was considered statistically significant.

\section{RESULTS}

\section{Baseline demographic characteristics}

Only 56 out of the 60 RA patients completed the full 12 weeks of intervention. The other patients (3 from placebo group, and 1 from SM group) withdrew during the trial due to dissatisfaction with treatment. Table 1 shows the baseline demographic characteristics of the RA patients. The values of various baseline demographic characteristics including age, gender, age at disease onset, duration of RA and body mass index (BMI) were similar in both groups.

Table 1: Baseline demographic characteristics of RA patients

\begin{tabular}{lcc}
\hline Variable & $\mathbf{S M}(\mathbf{n}=\mathbf{3 0})$ & Placebo $(\mathbf{n}=\mathbf{3 0})$ \\
\hline Age (years) & $45.7 \pm 1.5$ & $46.1 \pm 1.2$ \\
Gender & Male (12), Female (18) & Male (14), Female (16) \\
Age at disease onset (years) & $35.5 \pm 0.8$ & $36.1 \pm 0.9$ \\
Duration of RA treatment (days) & $4.8 \pm 0.3$ & $5.1 \pm 0.4$ \\
Body mass index (BMI; Kg/m $\left.\mathrm{m}^{2}\right)$ & $25.44 \pm 1.7$ & $25.10 \pm 1.2$ \\
\hline
\end{tabular}

Data are expressed as mean \pm standard deviation (SD). SM: Salvia miltiorrhiza 
ACR, GAD, HAQ and acute phase reaction proteins

Table 2 shows the ACR20/50 patient response and the related score/index in RA patients after SM and placebo treatments. The ARC 20 and ARC 50 responses of RA patients (30 and $13.3 \%$, respectively) were markedly improved ( $p$ $<0.01$ ) after 12 weeks of treatment with SM, when compared with the placebo group. Moreover, levels of the various ARC-related scores/indices such as GAD and $H A Q$, as well as acute phase reaction protein hs-CRP, were significantly decreased $(p<0.01)$ as a result of supplementation with $\mathrm{SM}$, relative to placebo. Level of VAS, Rh factor, DAS 28 and
inflammatory markers

As shown in Table 3, VAS, Rh factor (IgM) and DAS 28 were significantly reduced in RA patients after SM administration for 12 weeks, relative to placebo. Likewise, the levels of VAS, Rh factor $(\lg M)$ and DAS 28 were gradually decreased $(p<$ 0.01 ) in the SM group at the $12^{\text {th }}$ week, when compared with baseline values. Table 4 shows the levels of inflammatory markers (proinflammatory cytokines) in RA patients after SM and placebo treatments. The concentrations of all pro-inflammatory cytokines i.e. IL-1 $\beta$, II-6 and TNF- $\alpha$ were markedly lowered in RA patients in the SM group, when compared with the patients that received placebo $(p<0.01)$.

\section{Incidence of adverse effects}

Table 5 shows the incidence of various adverse effects in RA patients after SM and placebo treatments. During and after SM and placebo interventions, some minor adverse effects were observed. In the SM group, the adverse effects comprised headache, anemia, dizziness, nausea/vomiting, dyspepsia and skin rashes (although minimal). The adverse events seen in the placebo group were anemia, nausea/vomiting, dizziness, anorexia, headache and skin rashes/itching. Overall, anemia was the major adverse event noted in both groups (13.3 $\%$ in SM group, and $16.6 \%$ in placebo group). The other adverse events were minimal, implying the safety of SM.

Table 2: ACR (20\% and ACR 50\%) responses of RA patients and related scores/indices in RA patients after SM and placebo treatment

\begin{tabular}{lccc}
\hline Parameter & Duration & SM $(\mathbf{n}=\mathbf{3 0})$ & Placebo $(\mathbf{n}=\mathbf{3 0})$ \\
\hline ACR $(\%)$ in the $12^{\text {th }}$ week & ACR 20 & $9(30)$ & $2(6.66)$ \\
& ACR 50 & $4(13.3)$ & $1(3.33)$ \\
GAD score/index & Baseline & $80.5 \pm 2.5 \mathrm{a}$ & $81.0 \pm 2.7 \mathrm{a}$ \\
& $12^{\text {th }}$ week & $38.75 \pm 1.9 \mathrm{~b}^{\#}$ & $79.8 \pm 2.3 \mathrm{a}$ \\
$\mathrm{HAQ}$ & Baseline & $1.37 \pm 0.1 \mathrm{a}$ & $1.42 \pm 0.1 \mathrm{a}$ \\
& $12^{\text {th }}$ week & $0.85 \pm 0.1 \mathrm{~b}^{\#}$ & $1.35 \pm 0.1 \mathrm{a}$ \\
$\mathrm{hs}-\mathrm{CRP}(\mathrm{mg} / \mathrm{dL})$ & Baseline & $2.95 \pm 0.2 \mathrm{a}$ & $3.05 \pm 0.25 \mathrm{a}$ \\
& $12^{\text {th }}$ week & $1.58 \pm 0.1 \mathrm{~b}^{\#}$ & $3.10 \pm 0.30 \mathrm{a}$ \\
\hline
\end{tabular}

Data are expressed as mean \pm SD. For ACR, values are expressed as \% of patient response. Values within the same experimental group (SM or placebo) bearing different co-script letters are significantly different $(p<0.05)$. ${ }^{*} P<0.05 ;{ }^{\#} p<0.01$, SM vs placebo at the $12^{\text {th }}$ week: SM = Salvia miltiorrhiza; ACR = American College of Rheumatology; GAD = global assessment of disease; $\mathrm{HAQ}=$ Health assessment questionnaires; hs-CRP = high sensitivity $C$ reactive protein

Table 3: Pain scale (VAS), Rh factor and DAS 28 in RA patients after SM and placebo treatments

\begin{tabular}{lccc}
\hline Parameter & Duration & SM $(\mathbf{n}=\mathbf{3 0})$ & Placebo $(\mathbf{n}=\mathbf{3 0})$ \\
\hline Pain Scale (VAS) & Baseline & $78.80 \pm 5.20 \mathrm{a}$ & $80.10 \pm 6.70 \mathrm{a}$ \\
& $12^{\text {th }}$ week & $36.10 \pm 2.45 \mathrm{~b}^{\#}$ & $78.60 \pm 5.10 \mathrm{a}$ \\
$\operatorname{IgM}(\mathrm{g} / \mathrm{L})$ & Baseline & $1.89 \pm 0.12 \mathrm{a}$ & $1.82 \pm 0.14 \mathrm{a}$ \\
& 1 th $^{\text {week }}$ & $0.92 \pm 0.10 \mathrm{~b}^{\#}$ & $1.85 \pm 0.13 \mathrm{a}$ \\
$\mathrm{ESR}(\mathrm{mm} / \mathrm{h})$ & Baseline & $46.50 \pm 2.8 \mathrm{a}$ & $45.70 \pm 3.8 \mathrm{a}$ \\
& 1 th $^{\text {week }}$ & $25.80 \pm 1.6 \mathrm{~b}^{\#}$ & $44.90 \pm 3.5 \mathrm{a}$ \\
DAS 28 & Baseline & $6.25 \pm 0.5 \mathrm{a}$ & $6.19 \pm 0.6 \mathrm{a}$ \\
& $12^{\text {th }}$ week & $3.89 \pm 0.5 \mathrm{~b}^{\#}$ & $6.15 \pm 0.5 \mathrm{a}$ \\
\hline
\end{tabular}

Data are expressed as mean \pm SD. Values within the same experimental group (SM or placebo) bearing different co-script letters differ significantly $(p<0.05)$. ${ }^{*} P<0.05 ;{ }^{\#} p<0.01$, SM vs placebo at the $12^{\text {th }}$ week: SM $=$ Salvia miltiorrhiza; VAS = visual analogue score; lgG = immunoglobulin G; ESR = erythrocyte sedimentation rate; DAS $28=$ disease activity score 
Table 4: Levels of inflammatory markers (pro-inflammatory cytokines) in RA patients after SM and placebo treatments

\begin{tabular}{llcc}
\hline Parameter & Duration & SM $(\mathbf{n}=\mathbf{3 0})$ & Placebo $(\mathbf{n}=\mathbf{3 0})$ \\
\hline IL-1 $\beta(\mathrm{pg} / \mathrm{mL})$ & Baseline & $17.35 \pm 1.55 \mathrm{a}$ & $18.00 \pm 1.90 \mathrm{a}$ \\
& $12^{\text {th }}$ week & $8.60 \pm 1.01 \mathrm{~b}^{\#}$ & $17.20 \pm 2.05 \mathrm{a}$ \\
$\mathrm{IL}-6(\mathrm{pg} / \mathrm{mL})$ & Baseline & $38.00 \pm 4.40 \mathrm{a}$ & $37.68 \pm 3.20 \mathrm{a}$ \\
& $12^{\text {th }}$ week & $21.10 \pm 2.45 \mathrm{~b}^{\#}$ & $36.94 \pm 3.56 \mathrm{a}$ \\
TNF- $\alpha(\mathrm{pg} / \mathrm{mL})$ & Baseline & $122.95 \pm 10.65 \mathrm{a}$ & $121.50 \pm 11.39 \mathrm{a}$ \\
& $12^{\text {th }}$ week & $75.80 \pm 8.80 \mathrm{~b}^{\#}$ & $122.42 \pm 10.55 \mathrm{a}$ \\
\hline
\end{tabular}

Data are expressed as mean \pm SD. Values within the same experimental group (SM or placebo) bearing different subscript letters differ significantly $(p<0.05) .{ }^{*} P<0.05 ;{ }^{*} p<0.01, \mathrm{SM}$ vs placebo at the $12^{\text {th }}$ week: SM $=S a l v i a$ miltiorrhiza; IL-1 $\beta=$ interleukin 1 beta; IL-6 = interleukin 6; TNF- $\alpha=$ tumor necrosis factor-alpha; pg = picogram

Table 5: Incidence of various adverse effects in RA patients after SM and placebo treatments

\begin{tabular}{lcc}
\hline Adverse event & SM (n, \%) & Placebo (n, \%) \\
\hline Headache & $3(10)$ & $1(3.33)$ \\
Anemia & $4(13.3)$ & $5(16.6)$ \\
Dizziness & $2(6.66)$ & $3(10)$ \\
Nausea/vomiting & $2(6.66)$ & $4(13.3)$ \\
Dyspepsia & $1(3.33)$ & - \\
Anorexia & - & $3(10)$ \\
Diarrhea & - & - \\
Insomnia & - & $2(6.66)$ \\
Skin rashes/itching & $2(3.33)$ & $3(10)$ \\
\hline \multicolumn{2}{l}{ Data are expressed as } \\
\multicolumn{2}{l}{ Salvia miltiorrhiza } & \multicolumn{2}{l}{}
\end{tabular}

\section{DISCUSSION}

This is the first single-blind, placebo-controlled, randomized clinical trial conducted with SM alone (extract capsule) to assess the protective effect of SM through determination of ACR response and GAD, HAQ and DAS 28 scores, as well as levels of Rh factor, ESR, CRP and inflammatory cytokines in RA patients. The outcome of the trial showed that intake of SM capsule for 12 weeks markedly improved ACR 20/50 response of RA patients, with concomitant reductions in the levels of GAD, HAQ, DAS 28 scores, and decreases in the concentrations of $\mathrm{Rh}$ factor, ESR, CRP and inflammatory cytokines (markers). Patients in the placebo and SM groups showed similar baseline demographic characteristics. This helped to determine the impact of SM by comparing with a placebo group. The modified ACR classification criteria were developed in 2010 to plan a new set of criteria for diagnosing and classifying RA patients based on the various specifications which aid in early diagnosis and therapeutic intervention [18]. The ACR 20/50 patient response (positive improvement) represents a reduction of 20 or 50 $\%$ in the number of swollen (tender joints) in RA patients. Hence, ACR criteria and its response (ACR 20/50/70) are considered the primary outcomes of any anti-RA drug. In this study, RA subjects who ingested SM capsule for 12 weeks had significantly enhanced ACR20/50 patient response, relative to placebo-treated RA patients. Previously, Jie and his colleagues also demonstrated that treatment of RA patients with injection of salvia and complementary medicine considerably improved ACR 20/50 patient response [19]. During this clinical trial, there was no positive response in ACR70. Hence, it was not included. Furthermore, the ACR-related scores/indices such as GAD and HAQ were significantly lowered upon treatment with SM, when compared with the placebo group.

The levels of acute reaction protein hs-CRP and rheumatoid factors (Rh factors) are highly associated with joint damage especially during RA, Thus, hs-CRP and Rh factor (IgM) are clinically considered as markers for RA [20]. Significant decrease in the levels of hs-CRP, Rh and IgM were seen in the SM group (after 12 weeks, relative to baseline. Likewise, when compared to placebo on the $12^{\text {th }}$ week, hs-CRP and IgM levels were markedly decreased. A study on rabbits conducted by Zhang and his coworkers also reported that treatment with salvianolic acid and tanshinone (active components of SM) significantly lowered hs-CRP level [21]. In addition, pain scores (VAS and DAS-28 scores) were decreased by administration of SM capsule.

During RA, the pro-inflammatory cytokines IL$1 \beta / 6$ and TNF- $\alpha$ are exponentially synthesized (produced) by macrophages and lymphocytes, and they subsequently infiltrate the synovium to trigger inflammatory events involved in the pathophysiology of RA [22]. Similarly, in the present study, the baseline levels of the inflammatory markers IL- $1 \beta / 6$ and TNF- $\alpha$ in the placebo and SM groups were high, but on intervention with SM the levels of the inflammatory markers were markedly decreased. In the case of placebo, no difference was seen between values at baseline and values at the $12^{\text {th }}$ week. These results are in agreement with those reported by Xia and his co-workers, who also observed that treatment with salvianolic B (major phytocomponent of SM) significantly lowered the production of various pro- 
inflammatory cytokines i.e. IL-1 $1 \beta$, IL-6, and TNF$\alpha$ in a collagen-induced rheumatoid arthritis rat model [11].

The safety of SM was evaluated by checking various common adverse effects encountered during an anti-RA intervention. As mentioned earlier, in both groups, few minor adverse events were observed, especially headache, anemia, dizziness, nausea/vomiting, and skin rashes. However, the SM group showed more minimal levels of adverse effects than the placebo group. This shows that SM which has been widely used in TCM for many years, is safe. Moreover, these adverse events (anemia, headache and skin rashes) are common in RA patients. However, these adverse effects were noted, but they were lower in the SM group than in placebo group.

\section{Limitations of the study}

The major limitations of this study were the involvement of a small number of RA patients and the short duration of intervention (12 weeks). These might affect the interpretation of the results. In addition, the usage of SM (TCM) might make it harder to pinpoint the exact mechanism involved, since SM has many bioactive phytocomponents.

\section{CONCLUSION}

Based on the outcome of this single-blinded, placebo-controlled randomized clinical trial, SM may be recommended for combating $R A$ and its related symptoms via significant changes in ACR response and DAS 28 scores in RA patients, along with conventional anti-RA drugs. Nevertheless, in the future, there is need for a large-scale study of a longer duration which might give a more precise conclusion about the mechanism underlying the anti-RA effect of SM.

\section{DECLARATIONS}

\section{Acknowledgement}

This clinical trial was partially supported by Capital Medical University, Beijing, China.

\section{Conflict of interest}

No conflict of interest is associated with this study

\section{Contributions of authors}

We declare that this study was conducted by all the authors specified in this article and all are liabilities pertaining to claims related to this article will be borne by the authors. Zheng Yuan and Qiang Zhang both concepted and designed this study. Zhengrong Gao, Zheng Yuan and Changsong Zhao conducted this trial. Qiang Zhang and Zhengrong Gao drafted this manuscript. Changsong Zhao and Qiang Zhang involved in statistical analysis.

\section{Open Access}

This is an Open Access article that uses a funding model which does not charge readers or their institutions for access and distributed under the terms of the Creative Commons Attribution License (http://creativecommons.org/licenses/by/ 4.0) and the Budapest Open Access Initiative (http://www.budapestopenaccessinitiative.org/rea d), which permit unrestricted use, distribution, and reproduction in any medium, provided the original work is properly credited.

\section{REFERENCES}

1. Willich SN, Rossnagel $K$, Roll S, Wagner A, Mune $O$, Erlendson J, Kharazmi A, Sörensen H, Winther K. Rose hip herbal remedy in patients with rheumatoid arthritis-a randomised controlled trial. Phytomed 2010;17(2):87-93.

2. Lv QW, Zhang W, Shi Q, Zheng WJ, Li X, Chen H, Wu QJ, Jiang WL, Li HB, Gong L, Wei W. Comparison of Tripterygium wilfordii Hook $F$ with methotrexate in the treatment of active rheumatoid arthritis (TRIFRA): a randomised, controlled clinical trial. Ann Rheumatic Dis 2015;74(6):1078-1086.

3. Xu PG, Lei XF, Ren BD, Lv SY, Zhang JL. Diclofenac transdermal patch versus the sustained release tablet: $A$ randomized clinical trial in rheumatoid arthritic patients. Trop J Pharm Res 2017;16(2):477-482.

4. Chandran B, Goel A. A randomized, pilot study to assess the efficacy and safety of curcumin in patients with active rheumatoid arthritis. Phytother Res 2012; 26:1719-1725.

5. Klinkhoff A. Biological agents for rheumatoid arthritis. Drugs. 2004;64(12):1267-1283.

6. Venkatesha SH, Rajaiah R, Berman BM, Moudgil KD. Immunomodulation of autoimmune arthritis by herbal CAM. Evidence-Based Complement Altern Med 2011;2011.

7. Liu JJ, Wang XW, WU SF, WU QN, Hao DJ. Salvia miltiorrhiza aqueous root extract plays an important role in improving locomotor activity in rats with spinal cord injury. Trop J Pharm Res 2016;15(8):1667-1672.

8. Wang L, Ma R, Liu C, Liu H, Zhu R, Guo S, Tang M, Li Y, Niu J, Fu M, Gao S. Salvia miltiorrhiza: A potential red light to the development of cardiovascular diseases. Curr Pharm Des 2017;23(7):1077-1097.

Trop J Pharm Res, October 2020; 19(10): 2240 
9. Wang BQ. Salvia miltiorrhiza: Chemical and pharmacological review of a medicinal plant. J Med Plants Res 2010;4(25):2813-2820.

10. Liu QS, Luo $X Y$, Jiang $H$, Xing $Y$, Yang $M H$, Yuan $G H$, Tang Z, Wang $H$. Salvia miltiorrhiza injection restores apoptosis of fibroblast-like synoviocytes cultured with serum from patients with rheumatoid arthritis. Mol Med Rep 2015;11(2):1476-1482.

11. Xia ZB, Yuan YJ, Zhang QH, Li H, Dai JL, Min JK. Salvianolic acid $B$ suppresses inflammatory mediator levels by downregulating NF-KB in a rat model of rheumatoid arthritis. Med Sci Monit: Int Med JExp Clin Res 2018; 24:2524.

12. van Poppel PC, Breedveld P, Abbink EJ, Roelofs $H$, van Heerde $W$, Smits $P$, Lin W, Tan AH, Russel FG, Donders $R$, Tack CJ. Salvia miltiorrhiza root waterextract (danshen) has no beneficial effect on cardiovascular risk factors. A randomized double-blind cross-over trial. Plos One 2015;10(7):e0128695.

13. Salunkhe V, Papadopoulos $P$, Gutiérrez L. Culture of megakaryocytes from human peripheral blood mononuclear cells. Bioprotocol 2015;5:e1639.

14. Uhlig T, Haavardsholm EA, Kvien TK. Comparison of the Health Assessment Questionnaire (HAQ) and the modified HAQ (MHAQ) in patients with rheumatoid arthritis. Rheumatol 2005;45(4):454-458.

15. Wolfe $F$. Which $H A Q$ is best? A comparison of the $H A Q$, MHAQ and RA-HAQ, a difficult 8 item HAQ (DHAQ), and a rescored 20 item HAQ (HAQ20): analyses in 2,491 rheumatoid arthritis patients following leflunomide initiation. J Rheumatol 2001; 28(5): 982-989.
16. Prevoo ML, Van'T Hof M, Kuper HH, Van Leeuwen MA, Van De Putte LB, Van Riel PL. Modified disease activity scores that include twenty-eight-joint counts development and validation in a prospective longitudinal study of patients with rheumatoid arthritis. Arthritis Rheum: J Am College Rheumatol 1995;38(1):44-48.

17. Fransen J, Stucki G, van Riel PL. Rheumatoid arthritis measures: disease activity score (DAS), Disease activity score-28 (DAS28), Rapid assessment of disease activity in rheumatology (RADAR), and Rheumatoid arthritis disease activity index (RADAl). Arthritis Care Res: J Am College of Rheumatol 2003;49(S5):S214-24.

18. Kay J, Upchurch KS. ACR/EULAR 2010 rheumatoid arthritis classification criteria. Rheumatol. 2012;51(suppl_6):vi5-9.

19. Jie $L G$, Huang $Q C$, Shen $Y$, Sun WF, Wei $S, X u W$. Clinical observation of compound salvia injection in patients with active rheumatoid arthritis. Liaoning J Trad Chin Med2010; 10:42.

20. Plant MJ, Williams AL, O'Sullivan MM, Lewis PA, Coles $E C$, Jessop JD. Relationship between time-integrated $C$-reactive protein levels and radiologic progression in patients with rheumatoid arthritis. Arthritis Rheum: J Am College Rheumatol 2000;43(7):1473-1477.

21. Zhang FY, Hua SY, Fan YC. Effect of salvianolic acid $B$ and tanshinone IIA on the expression of the level of interleukin-8 and vascular cell adhesion molecule-1 in atherosclerosis rabbit models. J Shandong Univ Traditional Chinese Med 2006; 2:1-8.

22. Choy EH, Panayi GS. Cytokine pathways and joint inflammation in rheumatoid arthritis. New Engl J Med 2001;344(12):907-916. 